\title{
Evaluación del diagnóstico y notificación de Chikungunya en Ocaña, Colombia
}

\author{
Evaluation of the diagnosis \& reporting from the \\ Chikungunya in Ocaña, Colombia
}

Marco Antonio Márquez Gómez ${ }^{1}$, Lendy Andrea Escalante Angarita ${ }^{2}$.

\section{Resumen}

Objetivo. Evaluar el cumplimiento de los criterios diagnósticos y notificación de Chikungunya en Ocaña. Métodos. Estudio descriptivo y retrospectivo. Se revisaron 137 historias clínicas y sus fichas de notificación de Chikungunya al SIVIGILA, entre septiembre de 2014 y junio de 2015. Resultados. El número de casos de Chikungunya registrados hasta la fecha es bajo comparado con otras zonas del país. La calidad del diagnóstico del evento Chikungunya en Ocaña es deficiente, en términos del cumplimiento de los marcadores de la calidad del proceso de diagnóstico y notificación del evento.

Palabras clave: Aedes aegypti, Artralgia, Fiebre Chikungunya, Historia clínica, Ocaña, Salud pública, Vigilancia epidemiológica

\begin{abstract}
Objective. To assess the compliance of the diagnosis and reporting from the Chikungunya cases in Ocaña, Colombia. Methods. Descriptive and retrospective study. 137 medical records of Chikungunya were reviewed, as well as their notification records from the epidemiologic vigilance system "SIVIGILA", between September 2014 and June 2015. Results. The number of Chikungunya cases reported so far is low compared with other regions in the country. The quality of the diagnosis of the Chikungunya virus in Ocaña is deficient in terms of the compliance from the quality markers established for the diagnostic process as well as for the event reporting.
\end{abstract}

Keywords: Aedes aegypti, Arthralgia, Chikungunya Fever, Epidemiologic Surveillance, Medical Record, Ocańa, Public Health.

\footnotetext{
1. Químico Farmacéutico. Docente Escuela de Ciencias de la Salud, Universidad Nacional Abierta y a Distancia. Grupo de investigación TECNNOSALUD. 2. Enfermera. Enfermera vigilancia epidemiológica, Alcaldía Municipal de Ocaña. Grupo de investigación TECNNOSALUD.

Correspondencia: marco.marquez@unad.edu.co.

Fecha de recepción: Junio 27 de 2016 - Fecha de aprobación: Octubre 18 de 2016
} 


\section{Introducción}

El virus Chikungunya (CHIKV) es un arbovirus transmitido por mosquitos vectores del género Aedes; pertenece a la familia togaviridae (1-3) y al género de los alfa virus. Es un virus pequeño de 60 a $70 \mathrm{~nm}(1-2)$, posee membrana lipídica y cápside icosahédrica. Tiene genoma de ARN de banda simple con polaridad positiva, se adhiere a la superficie celular a través de receptores aún no identificados, y penetra a la célula blanco por endocitosis mediada por clatrina (1-2).

El virus Chikungunya fue aislado por primera vez en 1952 en Tanzania, África, con aparición de brotes esporádicos en humanos (2). Este país africano, ubicado en el trópico, tiene características similares, en lo ambiental y climático, a Colombia (4). El virus también se logró aislar entre los años 1960 y 1990 en países del Centro y Sur de África, incluyendo Sudán, Uganda, República Democrática del Congo, la República Centroafricana, Malaui, Zimbabue, Kenia y Sudáfrica, además de países africanos occidentales como Senegal, Benín, la República de Guinea, Costa de Marfil y Nigeria, donde alcanzó una sero-prevalencia de aproximadamente el $75 \%$ de la población, lo que constituyó un hecho de gran preocupación a nivel mundial (1-2).

Chikungunya significa, en la lengua kimakonde de Tanzania, "doblarse de dolor" (3), refiriéndose a la postura que adquieren los pacientes debido a los dolores articulares característicos de esta enfermedad (1).

La fiebre Chikungunya (CHIKF) se ha expandido explosivamente a varias regiones del mundo en las cuales no existía anteriormente y se ha convertido en una enfermedad emergente a nivel global (2), aumentado la preocupación y el interés respecto al impacto de este virus sobre la salud pública mundial (5).

En Colombia, el primer caso de Chikungunya se registró el 19 de julio de 2014, correspondiente a una mujer de 71 años procedente de República Dominicana. El 11 de septiembre de ése mismo año se notifica el primer caso autóctono confirmado por laboratorio correspondiente a un paciente proveniente del municipio de Mahates - Bolívar (6).

Ocaña es la segunda ciudad del departamento de Norte de Santander, ubicada en la cordillera oriental a 1202 msnm. Está situada a $8^{\circ} 14^{\prime} 15^{\prime \prime}$ de latitud Norte y $73^{\circ} 2$ '26" de longitud Oeste. Según el censo de 2005 con proyección a 2014, su población es de 97.479 habitantes, concentrándose el 90\% de la población en el casco urbano, el cual está dividido en 6 comunas (7).

Ocaña constituye un escenario propicio para la transmisión del virus y el consecuente desarrollo de la enfermedad de Chikungunya, ya que es zona endémica para dengue, presentando los mismos determinantes sociales que favorecen el desarrollo de la enfermedad (8): deficientes condiciones de salubridad, alta transmisión viral, altura por debajo de $2.200 \mathrm{msnm}$, necesidad de almacenamiento de agua, poca o nula educación en salud, baja percepción del riesgo, no uso de barreras protectoras, hacinamiento, migración, limitación del acceso a servicios de salud, poco entrenamiento en el diagnóstico y manejo de la enfermedad por parte del personal de salud, etc.

En el Departamento de Norte de Santander los primeros casos aparecieron registrados en el boletín epidemiológico No. 7 de fecha 21/09/2014, correspondiente a dos casos sospechosos en Cúcuta y San Calixto (9). El caso de Cúcuta correspondía a un caso importado confirmado y el caso de San Calixto se atendió en Ocaña, confirmándose por laboratorio en el mes de octubre (6).

Desde entonces y hasta el 30 de junio de 2015, fecha de corte del presente estudio, se han registrado en el municipio de Ocańa 137 casos (28 en 2014 y 109 en 2015), todos confirmados por clínica (10).

\section{Materiales y métodos}

Entre septiembre de 2014 y junio de 2015 se notificaron 137 casos de Chikungunya en el 
municipio de Ocaña. Con estos casos se realizó un estudio descriptivo, retrospectivo, en el cual se revisaron las historias clínicas de los 137 pacientes y las respectivas fichas de notificación epidemiológica reportadas al SIVIGILA. Cada caso se evaluó en dos dimensiones: la calidad de la notificación y el manejo clínico.

Para la evaluación de la calidad de la notificación se tomaron como variables de estudio la oportunidad en la notificación, la calidad del diligenciamiento de la ficha y la clasificación del caso (11).

Para la evaluación del manejo clínico se tomaron como variables de estudio una selección por conveniencia de aspectos relevantes del manejo del caso, los cuales se desarrollan en los lineamientos para la vigilancia de la enfermedad, expedidos por el Instituto Nacional de Salud (12). Estos aspectos son:

- Identificación en la historia clínica de los pacientes con factores de riesgo asociados (menor de un año, mayor de 65 años, mujer gestante, paciente con comorbilidad).

- Identificación en la historia clínica de los pacientes que residen o han visitado áreas epidémicas o endémicas por lo menos 15 días antes de la fecha de consulta.
- Identificación en la historia clínica de la triada de síntomas que caracterizan la enfermedad y la definen como caso: fiebre mayor de $38.5^{\circ} \mathrm{C}$, artralgias severas o artritis de comienzo agudo, rash cutáneo.

- Diagnóstico confirmado o presuntivo de Chikungunya.

- Registro en la historia clínica de la educación al paciente y su familia.

Se utilizó una hoja de cálculo de Microsoft Excel para el registro y análisis de la información. Los datos obtenidos fueron ordenados en distribuciones de frecuencia y presentados en tablas, los datos principales se midieron como número de casos, media y porcentaje.

\section{Resultados}

\section{Aspectos generales}

Se revisaron 137 historias clínicas y fichas de notificación del evento Chikungunya. La edad promedio de los pacientes fue de 26 años, con mínimo de 2 y máximo de 74 . El 33\% de los pacientes eran hombres, mientras que el 67\% eran mujeres, Tabla 1 .

Tabla 1. Casos notificados de Chikungunya Ocaña 2014 - 2015. Distribución por rango de edad y sexo

\begin{tabular}{|c|c|c|c|}
\hline \multirow{2}{*}{ Rango de edad } & \multicolumn{2}{|c|}{ Sexo } & \multirow{2}{*}{ Total } \\
\hline & Hombre & Mujer & \\
\hline 1 a 5 años & 5 & 3 & 8 \\
\hline 6 a 10 años & 4 & 5 & 9 \\
\hline 11 a 15 años & 3 & 5 & 8 \\
\hline 16 a 20 años & 4 & 14 & 18 \\
\hline 21 a 25 años & 4 & 17 & 21 \\
\hline 26 a 30 años & 4 & 5 & 9 \\
\hline 31 a 35 años & 2 & 6 & 8 \\
\hline 36 a 40 años & 7 & 11 & 18 \\
\hline 41 a 45 años & 4 & 8 & 12 \\
\hline 46 a 50 años & 3 & 8 & 11 \\
\hline 51 a 55 años & 2 & 3 & 5 \\
\hline 56 a 60 años & 2 & 3 & 5 \\
\hline 61 a 65 años & 0 & 1 & 1 \\
\hline 66 a 70 años & 0 & 1 & 1 \\
\hline 71 a 75 ańos & 1 & 2 & 3 \\
\hline TOTAL & 45 & 92 & 137 \\
\hline
\end{tabular}

Fuente. Cálculos de los autores. 
El 96\% de los pacientes estaban afiliados a una EPS, mientras que el $4 \%$ restante no tenían cobertura. En cuanto a régimen de afiliación, el 56\% estaba afiliado al régimen subsidiado, el $41 \%$ al régimen contributivo y el 3\% al régimen especial.

El 79\% de los pacientes provenían del casco urbano del municipio Ocańa, el $2 \%$ provenían de la zona rural y el $19 \%$ restante provenían de otros municipios.

El número de casos notificados pasó de unos pocos casos entre septiembre y noviembre de 2014, a un incremento considerable durante los meses de diciembre de 2014 y enero de 2015 (20 y 33 respectivamente), para después estabilizarse a un promedio de 15 casos por mes. Cinco IPS, las cuales se identifican con su código de prestador, notificaron el 100\% los casos estudiados, Tabla 2.

\section{Calidad de la notificación}

Todos los eventos fueron reportados a la unidad notificadora municipal el mismo día de consulta del paciente y todas las fichas de notificación se diligenciaron completa y correctamente. La clasificación inicial del caso se realizó conforme a los lineamientos establecidos por el Instituto Nacional de Salud (11). No obstante, se encontraron diferencias entre lo notificado y lo diagnosticado: solo 48 historias correspondientes al 35\% de los casos tenían diagnóstico confirmado o presuntivo de Chikungunya.

\section{Calidad del manejo clínico}

Se identificaron 7 pacientes con factores de riesgo, de los cuales 4 pacientes tenían edades superiores a 65 ańos y 3 mujeres en estado de embarazo.

En total 30 médicos atendieron los 137 casos. El promedio global de cumplimiento de los criterios de diagnóstico y manejo del caso fue del $42 \%$ para todas las historias revisadas, Tabla 3.

Se identificó una sola historia clínica en la cual se registraron los seis criterios diagnósticos escogidos como marcadores de calidad; en contraste, cuatro historias no contaban con ningún criterio. El 78\% de las historias solo identifican entre cero y tres criterios diagnósticos, Tabla 4.

Tabla 2. Número de casos de Chikungunya notificados por IPS y mes, Ocaña, 2014 a 2015

\begin{tabular}{|c|c|c|c|c|c|c|}
\hline \multirow{2}{*}{ Mes } & \multicolumn{5}{|c|}{ IPS que notifica } & \multirow{2}{*}{ Total } \\
\hline & 0054701 & 0060601 & 0068601 & 0144501 & 0192801 & \\
\hline Septiembre 2014 & 1 & 0 & 0 & 0 & 0 & 1 \\
\hline Octubre 2014 & 4 & 0 & 0 & 0 & 0 & 4 \\
\hline Noviembre 2014 & 0 & 3 & 0 & 0 & 0 & 3 \\
\hline Diciembre 2014 & 3 & 16 & 1 & 0 & 0 & 20 \\
\hline Enero 2015 & 22 & 5 & 5 & 1 & 0 & 33 \\
\hline Febrero 2015 & 11 & 1 & 0 & 0 & 1 & 13 \\
\hline Marzo 2015 & 11 & 3 & 0 & 0 & 0 & 14 \\
\hline Abril 2015 & 15 & 1 & 0 & 0 & 0 & 16 \\
\hline Mayo 2015 & 19 & 1 & 0 & 0 & 0 & 20 \\
\hline Junio 2015 & 13 & 0 & 0 & 0 & 0 & 13 \\
\hline Total & 99 & 30 & 6 & 1 & 1 & 137 \\
\hline Porcentaje & $72 \%$ & $22 \%$ & $4 \%$ & $1 \%$ & $1 \%$ & $100 \%$ \\
\hline
\end{tabular}

Fuente. Cálculos de los autores. 
Tabla 3. Porcentaje de cumplimiento de criterios diagnósticos y médicos que notifican casos de Chikungunya, Ocaña. 2014 a 2015.

\begin{tabular}{|cccc|}
\hline IPS que notifica & $\begin{array}{c}\text { Número de médicos } \\
\text { que notifican }\end{array}$ & $\begin{array}{c}\text { Número de casos } \\
\text { notificados }\end{array}$ & $\begin{array}{c}\text { Porcentaje de } \\
\text { cumplimiento }\end{array}$ \\
\hline 0068601 & 3 & 6 & $61 \%$ \\
0060601 & 8 & 30 & $58 \%$ \\
\hline 0054701 & 17 & 99 & $36 \%$ \\
\hline 0192801 & 1 & 1 & $33 \%$ \\
\hline 0144501 & 1 & 1 & $33 \%$ \\
\hline TOTAL & $\mathbf{3 0}$ & $\mathbf{1 3 7}$ & $\mathbf{4 2 \%}$ \\
\hline
\end{tabular}

Fuente. Cálculos de los autores.

Tabla 4. Número de criterios diagnósticos identificados por IPS que notifica, Ocańa. 2014 a 2015.

\begin{tabular}{|c|c|c|c|c|c|c|c|c|}
\hline \multirow{2}{*}{ IPS que notifica } & \multicolumn{7}{|c|}{ Número de criterios de diagnóstico identificados } & \multirow{2}{*}{ Total } \\
\hline & 6 & 5 & 4 & 3 & 2 & 1 & 0 & \\
\hline 0068601 & 0 & 3 & 0 & 2 & 0 & 1 & 0 & 6 \\
\hline 0060601 & 1 & 4 & 10 & 10 & 4 & 1 & 0 & 30 \\
\hline 0054701 & 0 & 1 & 10 & 20 & 45 & 19 & 4 & 99 \\
\hline 0192801 & 0 & 0 & 0 & 0 & 1 & 0 & 0 & 1 \\
\hline 0144501 & 0 & 0 & 0 & 0 & 1 & 0 & 0 & 1 \\
\hline TOTAL & 1 & 8 & 20 & 32 & 51 & 21 & 4 & 137 \\
\hline Porcentaje & $1 \%$ & $6 \%$ & $15 \%$ & $23 \%$ & $37 \%$ & $15 \%$ & $3 \%$ & $100 \%$ \\
\hline
\end{tabular}

Fuente. Cálculos de los autores.

El rash cutáneo fue el síntoma mayormente identificado con $75 \%$, le siguen en su orden, artralgia con $72 \%$ y fiebre con $38 \%$.

En solo 37 casos, equivalentes al 27\%, se registró que se indagó sobre la procedencia del paciente.

La educación al paciente y su familia solo se registró en 6 casos (4\%); distribuidos en tres casos notificados por 006801; dos casos notificados por 0060601 y uno notificado por 0054701 .

\section{Discusión}

A pesar de algunas predicciones sobre la diseminación y el carácter epidémico de la enfermedad debido a su alta tasa de ataque (1$2,5-6,13)$, en el municipio de Ocaña no se ha presentado una explosión dramática de casos como sí ha ocurrido en otras regiones del país (10), a pesar de ser un área endémica para dengue (8), enfermedad que comparte el mismo vector de la fiebre Chikungunya, una alta movilidad geográfica $(7,13)$, y de poseer los determinantes sociales que favorecen el desarrollo de la enfermedad $(3,13)$.

El segmento de población más afectado fue el de 21 a 25 ańos, con 21 casos, de los cuales 17 eran mujeres. En general, la mayoría de las mujeres encuestadas son amas de casa y permanecen en sus hogares, lo que explica el mayor índice de infección en este segmento de la población, debido a los hábitos diurnos del mosquito y a reproducirse en agua almacenada en los hogares (8).

La escasa correspondencia entre el diagnóstico y la notificación en el 35\% de los casos estudiados, permite inferir que existen deficiencias tanto en la 
clasificación de los casos como en el reporte de estos al sistema de vigilancia epidemiológica; situación que ya ha sido evidenciada en otros estudios (1415).

El cumplimiento de los criterios determinados como marcadores de la calidad del diagnóstico de los casos de Chikungunya (12) revela una sorprendente asimetría en el manejo de los casos: por un lado, la variabilidad del cumplimiento entre instituciones, pero también, entre profesionales médicos:

- Se diagnostica un caso de Chikungunya en el cual se identifica un solo criterio.

- No se diagnostican como Chikungunya cuatro casos que reúnen cuatro criterios.

- Médicos que atendieron más de cinco casos tienen diferentes porcentajes de identificación de criterios diagnósticos.

- Se esperarían mejores diagnósticos al final de la línea de tiempo, pero se encontraron porcentajes de cumplimientos bajos (menores de $50 \%$ ) en todos los meses de estudio.

Lo anterior puede explicarse por una combinación de falta de entrenamiento o desconocimiento de los lineamientos de diagnóstico de la enfermedad, y por la aversión de algunos profesionales médicos al manejo de enfermedades que constituyen eventos de interés en salud pública. Esta situación junto a la posible delegación del diligenciamiento de la ficha de notificación son solo juicios empíricos de los autores formulados a la luz de los datos analizados; por tanto, la indagación sobre sus posibles causas, será un imperativo de continuidad del presente estudio.

La fiebre como síntoma de Chikungunya, puede llevar a un diagnóstico diferencial (12-13) y por consiguiente requiere de su medición. El 38\% de los casos con fiebre registrada corresponde al cumplimiento del criterio clínico, es decir, que el paciente haya presentado fiebre superior a $38.5^{\circ} \mathrm{C}$.
No obstante en la revisión de las historias clínicas se pudo constatar que en un alto número de historias no se registró la temperatura del paciente. La mayor identificación del rash y la artralgia puede deberse a que son signos y síntomas fáciles de observar, mientras que la temperatura implica la intervención del médico o de la enfermera para su medición.

El acto de brindar educación al paciente y su familia se incluyó como criterio de calidad debido a que hace parte integral de la atención al paciente (12). Las historias clínicas revisadas revelan que solo en seis casos se brindó educación al paciente, sin precisar sus contenidos ni alcances; lo que evidencia, desde el punto de vista ético, un escaso o nulo compromiso del personal asistencial en el manejo adecuado de la enfermedad que permita prevenir su ocurrencia y/o mitigar su impacto (6).

El número de casos de Chikungunya registrados hasta la fecha en el municipio es considerablemente bajo en comparación con otras zonas del país (10). No obstante, se requiere una mayor preparación del personal de salud para el diagnóstico oportuno y el manejo adecuado de los pacientes; y por sobre todo, una acción eficaz y continua de las autoridades civiles, principalmente en educación a la comunidad, para evitar la proliferación del mosquito y la transmisión del virus.

La calidad del diagnóstico del evento Chikungunya en el municipio de Ocaña es deficiente, en términos del cumplimiento de los criterios convenidos como marcadores de la calidad del proceso de diagnóstico y notificación (12). Es indispensable cumplir con los lineamientos para el manejo de la enfermedad (6).

Es compromiso del personal médico mejorar sus habilidades y entrenamiento en el manejo de la enfermedad para lograr un diagnóstico correcto y por ende un manejo eficaz del evento. Se requiere que las instituciones de salud del municipio realicen intervenciones en aras de propiciar un mayor compromiso del médico con la prevención, la identificación, el diagnóstico, el manejo y la 
notificación de los eventos de Chikungunya, que son eventos de interés en salud pública (6).

\section{Agradecimientos}

Los autores expresan su agradecimiento a la Universidad Nacional Abierta y a Distancia, a la Secretaria de Desarrollo Humano de la Alcaldía del municipio de Ocaña, y a Carolina Gómez Márquez, por su contribución a la realización de este trabajo.

\section{Referencias}

1. Corrales-Aguilar E., Troyo A., Calderón-Arguedas Ó. Chikungunya: un virus que nos acecha. Acta Méd. Costarric. 2015 ene. (57): 7-15. Disponible en: http://www.redalyc.org/ articulo.oa?id $=43433759002$

2. Martínez Fernández L., Torrado Navarro Y. P. Fiebre Chikungunya. Rev cubana med. 2015 Mar; 54(1): 74-96. Disponible en: http://scielo.sld.cu/scielo.php?script=sci_ arttext\&pid=S0034-75232015000100008\&lng=es

3. República Dominicana. Ministerio de Salud Pública. Guía de manejo clínico para la infección por el virus Chikungunya. Rev Pediatr Aten Primaria. 2014 Sep.; 16(63): e119 - e119. Disponible en: http://scielo.isciii.es/scielo.php?script=sci_ arttext\&pid=S1 13976322014000400023\&lng=es

4. Salazar-Ceballos A, Álvarez-Miño L. Asociación entre Factores Climatológicos y Tasa de Incidencia del Dengue en Santa Marta, Colombia, 2007-2013. Rev Ciencias Biomédicas 01/2014; 5(1):41-47 Disponible en: http://revistas.unicartagena.edu.co/ index.php/cienciasbiomedicas/article/download/1200/1110

5. Organización Mundial de la Salud-OMS/Organización Panamericana de la Salud- OPS. Preparación y respuesta ante la eventual introducción del virus chikungunya en las Américas. Washington D.C. 2011. Disponible en: http://www1.paho. org/hq/dmdocuments/CHIKV_Spanish.pdf

6. Martínez, M.; Gómez, S. Chikungunya en Colombia, el inicio de la transmisión autóctona, 2014. Informe Quincenal Epidemiológico Nacional. [PDF] 2014; 19 (18): Pág. 260 a 279. Disponible en: http://www.ins.gov.co/iqen/IQUEN/ IQEN\%20vol\%2019\%202014\%20num\%2018.pdf

7. Alcaldía municipal de Ocaña. Plan de Desarrollo 2012 - 2015 Ocańa Confiable y Participativa. [Internet]. Disponible en: http://ocana-nortedesantander.gov.co/apc-aa-fil es/38656632356330656332656230383763/ACUERDO_
PLAN_DE_DESARROLLO_2012_2015_OCA_A_PARTICIPATIVA_Y_CONFIABLE_2.pdf

8. Instituto Nacional de Salud. Informe final Dengue, Colombia 2014. 2014. [PDF]. Disponible en: http://www.ins.gov.co/ lineas-de-accion/Subdireccion-Vigilancia/Informe $\% 20 \mathrm{de} \% 20$ Evento\%20Epidemiolgico/Dengue\%202014.pdf

9. Instituto Nacional de Salud. Boletín Epidemiológico evento prioritario: Chikungunya No. 7. 2014 sep. [PDF]. Disponible en: http://www.ins.gov.co/lineas-de-accion/SubdireccionVigilancia/informacion-epidemiologica/BoletinChikungunya/ INS\%20Boletin\%20Diario\%20No\%207\%20CHIK.pdf

10. Instituto Nacional de Salud. Resumen Chikungunya a semana 25 de 2015. [Internet]. Disponible en: http:// www.ins.gov.co/Noticias/Chikungunya/Resumen $\% 20$ Chikungu\%C3\%B1a\%20SEMANA\%2025\%202015.pdf

11. Instituto Nacional de Salud. Lineamientos 2015 para la prevención, vigilancia y control en salud pública. [Internet]. Disponible en: http://www.ins.gov.co/lineas-deaccion/Subdireccion-Vigilancia/Lineamientos $\% 20 y \% 20$ Documentos/01\%20LINEAMIENTOS\%202015.pdf

12. Instituto Nacional de Salud. Lineamientos para el manejo clínico de los pacientes con el virus Chikungunya (CHIKV). 2014 sep. [PDF]. Disponible en: https://www.minsalud. gov.co/sites/rid/Lists/BibliotecaDigital/RIDE/VS/PP/ET/ Anexo\%204\%20Lineamientos\%20\%20para\%20el\%20 manejo $\% 20 \mathrm{cl} \% \mathrm{C} 3 \%$ ADnico $\% 20 \mathrm{de} \% 20$ pacientes $\% 20$ CHIKV\%20\%202014.pdf

13. Placeres Hernández J. F., Martínez Abreu J., Chávez González L., Rodríguez E., de León Rosales L. Fiebre causada por el virus Chikungunya, enfermedad emergente que demanda prevención y control. Rev. Med. Electrón. 2014 Oct; 36(5): 596609. Disponible en: http://scielo.sld.cu/scielo.php?script=sci_ arttext\&pid=S1684-18242014000500007\&lng=es

14. Moreno Gómez G. A., Moreno Gómez J. G., Cabezas Restrepo Á. M., Múnera Benavides J. E., Ocampo Alzate K. Pa., Moreno Villegas V. Cumplimiento en la notificación de casos probables de dengue en el Área Metropolitana Centro Occidente de Colombia en el año 2014. Revista médica Risaralda. 2016 Jul; 22(2): 75-78. Disponible en: http://www.scielo.org.co/scielo.php?script=sci_ arttext\&pid=S0122-06672016000200002\&lng=en

15. Millan J, Yunda L. An Open-Access Web-based medical image atlas for collaborative Medical image sharing, processing, Web Semantic searching and analysis with uses in medical training, research and second opinion of cases. Nova. 2014;12(22):14350 